\title{
Seasonal variations in brightness temperature for central Antarctica
}

\author{
C. J. VAN DER VEen AND K. C. JeZEK \\ Byrd Polar Research Center, The Ohio State University, Columbus, OH 43210, U.S.A.
}

\begin{abstract}
The radiative-transfer model developed by Zwally (1977) is modified and coupled to a one-dimensional time-dependent temperature model, to calculate the seasonal variation in brightness temperature. By comparing this with observed records, the radiative properties of firn can be determined. By retaining scattering as a source term in the radiative transfer function, agreement between model-derived scattering and absorption coefficients and those calculated from the $\mathrm{Mie} /$ Rayleigh scattering theory can be obtained. The horizontal brightness temperature is not linked to the vertical one through a constant power reflection coefficient.
\end{abstract}

\section{INTRODUCTION}

Patterns of spatially varying microwave brightness temperature across the polar ice sheets have intrigued glaciologists with the prospect of deducing the extent and degree of surface melt, accumulation rate, and physical temperature (Zwally and Gloersen, 1977; Jezek and others, 1990). While that objective remains elusive, considerable progress has been made in developing a theoretical framework for modelling observed brightness temperature at least at a few locations (Chang and others, 1976, 1980; Zwally, 1977; Comiso and others, 1982).

In this paper, we concentrate our analysis on the seasonal variation in brightness temperature averaged over a sector of the East Antarctica ice sheet. Coupling a radiative-transfer model with a numerical calculation of temperature at depth, and using recent estimates of firn dielectric properties, we investigate the role of the Antarctic asymmetric seasonal temperature cycle on the brightness temperature cycle measured at 18-19 and $37 \mathrm{GHz}$. We also investigate the reasons for differences in the maximum summer and minimum winter brightness temperatures observed at these frequencies. The radiative-transfer model developed by Zwally (1977) is modified to obtain a better agreement betweeen radiative parameters determined by matching calculated brightness temperatures to observed ones, and the theoretically as well as experimentally-determined radiative parameters.

\section{TEMPERATURE MODEL}

The model used to calculate the temperature evolution in the upper firn layer is essentially that of Greuell and Oerlemans $(1986,1989)$. In short, horizontal advection of heat is neglected and the one-dimensional time-depen-

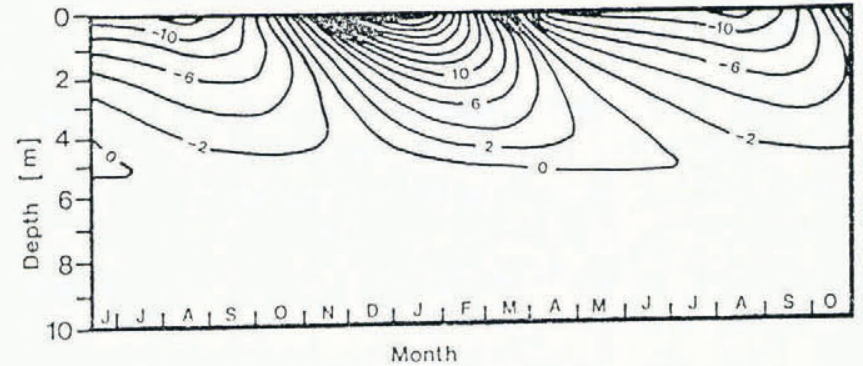

Fig. 1. Calculated evolution of the difference between temperature at depth and the annual-mean surface temperature for Vostok Station. Contour interval is $2 \mathrm{~K}$.

dent energy equation is solved. Radiative fluxes at the surface are computed from the solar zenith angle, surface albedo, cloudiness, and (calculated) temperature of the snow surface. Sensible and latent heat fluxes are calculated from standard meteorological variables. For Vostok Station in East Antarctica, multi-year monthly mean values of climate parameters are compiled by Schwerdtfeger (1970). These data are used here with the understanding that the monthly mean values are representative for the mid-month climate, so that intermediate values can be obtained by interpolation.

Results of the temperature calculation are shown in Figure 1. Because vertical exchange of heat in the firn is a diffusive process, the amplitude of the downward traveling temperature wave associated with the summer warming decreases rapidly with depth. At about $10 \mathrm{~m}$ below the surface, the annual cycle has virtually disappeared, as predicted by theoretical models. At $5 \mathrm{~m}$ below the surface, the time lag between the temperature maximum and the summer maximum at the surface is almost 4.5 months. At the surface, the annual march in 
temperature exceeds that of the atmosphere as a result of the additional heating of the surface by solar radiation.

\section{RADIATIVE-TRANSFER MODEL}

The observed microwave radiation of the polar ice sheets originates not only from the suface, but from within the firn layer as well. Folllowing Zwally (1977), the brightness temperature of the ice surface is calculated from the depth integral of the physical temperature at depth, times the emissivity at depth, weighted by a radiative transfer function. Zwally (1977) arrives at the following expression

$$
T_{\mathrm{B}}=\int_{0}^{\infty} \gamma_{\mathrm{a}} \exp \left[-\left(\gamma_{\mathrm{so}}+\gamma_{\mathrm{a}}\right) z-\frac{s z^{2}}{2}\right] T(z) \mathrm{d} z,
$$

where

$$
\begin{aligned}
& T_{\mathrm{B}}=\text { brightness temperature } \\
& \gamma_{\mathrm{a}}=\text { absorption coefficient } \\
& \gamma_{\mathrm{so}}=\text { scattering coefficient at the surface } \\
& s=\text { linear increase of scattering with depth } \\
& z \quad=\text { depth below the surface, measured vertically. }
\end{aligned}
$$

In deriving Equation (1), Zwally (1977) assumed that scattering is small relative to absorption and emission, in order to obtain an analytical solution. However, as acknowledged by Zwally, this assumption is not really valid so that radiative parameters determined with this equation may be erroneous. This assumption need not be imposed if the full source term is preserved in the radiative transfer function (Zwally, 1977, equation (26)), which results in:

$$
T_{\mathrm{B}}=\int_{0}^{\infty} \gamma_{\mathrm{a}}[1+Z] \exp \left[-\left(\gamma_{\mathrm{so}}+\gamma_{\mathrm{a}}\right) z-\frac{s z^{2}}{2}\right] T(z) \mathrm{d} z .
$$

As inspection of the equations given by Zwally (1977) shows, the correction factor, $Z$, is a complex function of temperature, depth and wavelength of the radiation. As a start, however, this term may be included by neglecting its temperature and depth variation. In that case, the expression for the brightness temperature can be written as

$$
T_{\mathrm{B}}=\int_{0}^{\infty} \Gamma_{\mathrm{a}} \exp \left[-\left(\Gamma_{\mathrm{so}}+\Gamma_{\mathrm{a}}\right) z-\frac{s z^{2}}{2}\right] T(z) \mathrm{d} z,
$$

where

$$
\Gamma_{\mathrm{a}}=\gamma_{\mathrm{a}}(1+Z)
$$

denotes the modified absorption coefficient, and

$$
\Gamma_{\mathrm{so}}=\gamma_{\mathrm{so}}-Z \gamma_{\mathrm{a}}
$$

denotes the modified scattering coefficient. The net loss of radiation near the surface is given by the sum of these two coefficients

$$
\begin{aligned}
\Gamma_{\mathrm{eo}} & =\Gamma_{\mathrm{so}}+\Gamma_{\mathrm{a}} \\
& =\gamma_{\mathrm{so}}+\gamma_{\mathrm{a}}
\end{aligned}
$$

which is independent of the value of $Z$, irrespective of how $Z$ varies with depth. In the approximation $Z \approx 0$, the modified coefficients equal the true absorption and scattering coefficients.

If the observing satellite images the ice sheet obliquely, a correction for the angle of observation needs to be made. Instead of radiation originating from directly below, the satellite senses radiation that has travelled at an angle $\theta_{2}$ through the firn. This angle is related to the angle of observation, $\theta_{1}$, according to Snell's law. The modified version of Equation (3) becomes

$$
\begin{aligned}
& T_{\mathrm{B}}= \\
& \int_{0}^{\infty} \Gamma_{\mathrm{a}} \exp \left[\left[-\left(\Gamma_{\mathrm{so}}+\Gamma_{\mathrm{a}}\right) z-\frac{s z^{2}}{2}\right] \sec \Theta_{2}\right] T(z) \sec \Theta_{2} \mathrm{~d} z .
\end{aligned}
$$

To account for reflection of the upwelling radiation at the surface this expression needs to be multiplied by (1 $R_{\mathrm{p}}$ ), where $R_{\mathrm{p}}$ represents the power reflection coefficient and is polarization dependent.

The radiative-transfer model used to calculate the brightness temperature contains four unknown parameters, namely the modified absorption coefficient $\Gamma_{\text {a }}$, the modified scattering coefficient at the surface $\Gamma_{\text {so }}$, the linear increase of scattering with depth $s$, and the power reflection coefficient $R_{\mathrm{p}}$. The value of $s$ has much less effect on calculated brightness temperatures than the values of $\Gamma_{\mathrm{a}}$ and $\Gamma_{\mathrm{so}}$, and in the present calculations, the intermediate value $s=0.015 \mathrm{~m}^{-1}$ (Zwally, 1977) is used. For the vertically-polarized channels, the power reflection coefficient is near zero and the remaining two parameters can be determined by comparing the calculated seasonal variation in brightness temperature with the observed variation. The power reflection coefficient for the horizontally-polarized channels can then be estimated by applying these values to the horizontal channels as well.

Calculated curves of brightness temperature throughout the year can be characterized by two quantities, namely the minimum brightness temperature, $T_{\mathrm{Bmin}}$, and the difference between the maximum and minimum,

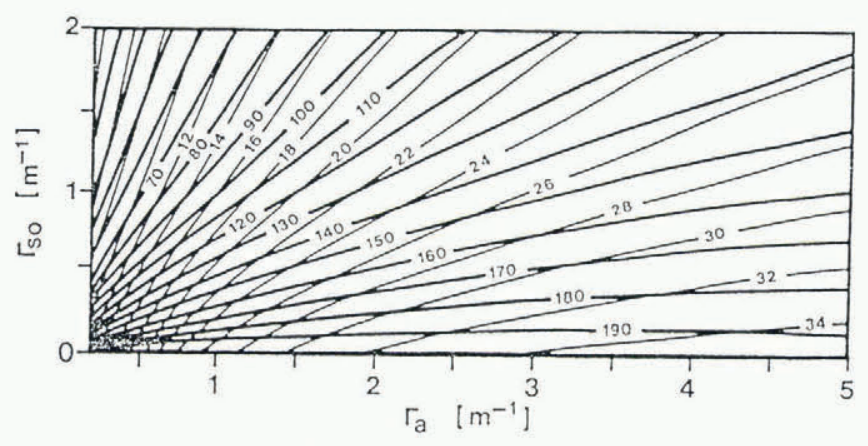

Fig. 2. Contour diagram showing the relation between calculated minimum brightness temperature (heavy curves, contour interval $10 \mathrm{~K}$ ) and amplitude of the seasonal swing (light curves, contour interval $2 K$ ), and the radiative parameters, $\Gamma_{\mathrm{a}}$ (modified absorption coefficient) and $\Gamma_{\text {so }}$ (modified scattering coefficient at the surface). The angle of observation is $\Theta_{1}=49^{\circ}\left(\Theta_{2}=32^{\circ}\right)$ and the firn temperatures shown in Figure 1 are used. 
$\Delta T_{\mathrm{B}}$. Because $\Gamma_{\mathrm{a}}$ and $\Gamma_{\mathrm{so}}$ affect the minimum brightness temperature and the seasonal variation in a different manner, any combination of $T_{\mathrm{Bmin}}$ and $\Delta T_{\mathrm{B}}$ unambiguously determines the two radiative coefficients, and vice versa. This is illustrated by the contour diagram shown in Figure 2.

Absorption and scattering at the surface strongly affect both $T_{\mathrm{Bmin}}$ and $\Delta T_{\mathrm{B}}$. As absorption increases (larger value of $\Gamma_{\mathrm{a}}$ ), the brightness temperature also increases. This is because the firn is assumed to be in thermal equilibrium so that when the absorption of a layer contribution to the brightness temperature also increases.

For high absorption coefficients the amplitude of the seasonal variation in $T_{\mathrm{B}}$ is largest because the emitted radiation originates from the near-surface layer. For small absorption, deeper layers contribute more significantly to the calculated brightness temperature. But, as shown in Figure 1, there is a considerable time lag between the temperature maximum at the surface and that at depth.

Finally, increased scattering reduces both the brightness temperature and the seasonal amplitude. The reason for this is that the optical depth decreases (linearly) with the scattering coefficient.

\section{APPLICATION TO VOSTOK STATION}

Two data sets are available to test the present model. The first is derived from the Scanning Multichannel Microwave Radiometer (SMMR) launched on the Nimbus 7 satellite in 1977. Jezek and others (1990) use the 8 year record of observations to construct time series of brightness temperature over Antarctica. They adopt the hypothesis that specific glacial regimes exhibit characteristic microwave signatures, and divide the ice sheet into five spatial regimes. Data within each regime are averaged spatially and temporally to obtain monthly mean values. For the present study, the series of monthly mean values for the Ice Dome region (which includes Vostok Station) is further averaged to find the long-term averaged (1979-85) monthly mean values of brightness temperature. Data for the 18 and $37 \mathrm{GHz}$ vertical and horizontal channels are used here, but corrected to account for relative calibration differences between the SMMR and SSM/I data (Jezek and others, 1991).

Data obtained by the Navy's Special Sensor Microwave Imager (SSM/I) are also used in this study. Time series of SSM/I brightness temperature for the 19.35 and
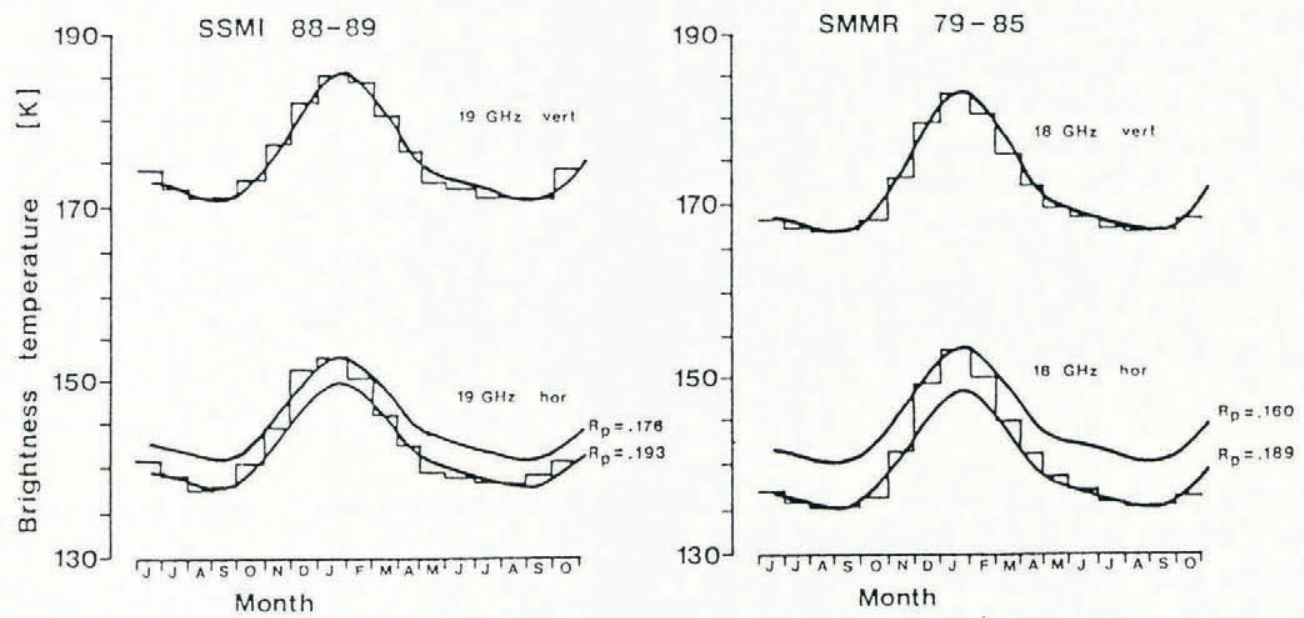

Fig. 3. Calculated (heavy curves) and measured (stepped curves) brightness temperature for the $18 \mathrm{GHz} S M M R$ (right) and $19 \mathrm{GHz}$ SSM/I (left) vertical (upper panel) and horizontal (lower panel) channels. The horizontal brightness temperature is calculated from the vertical one using expression (8) with a constant power reflection coefficient.
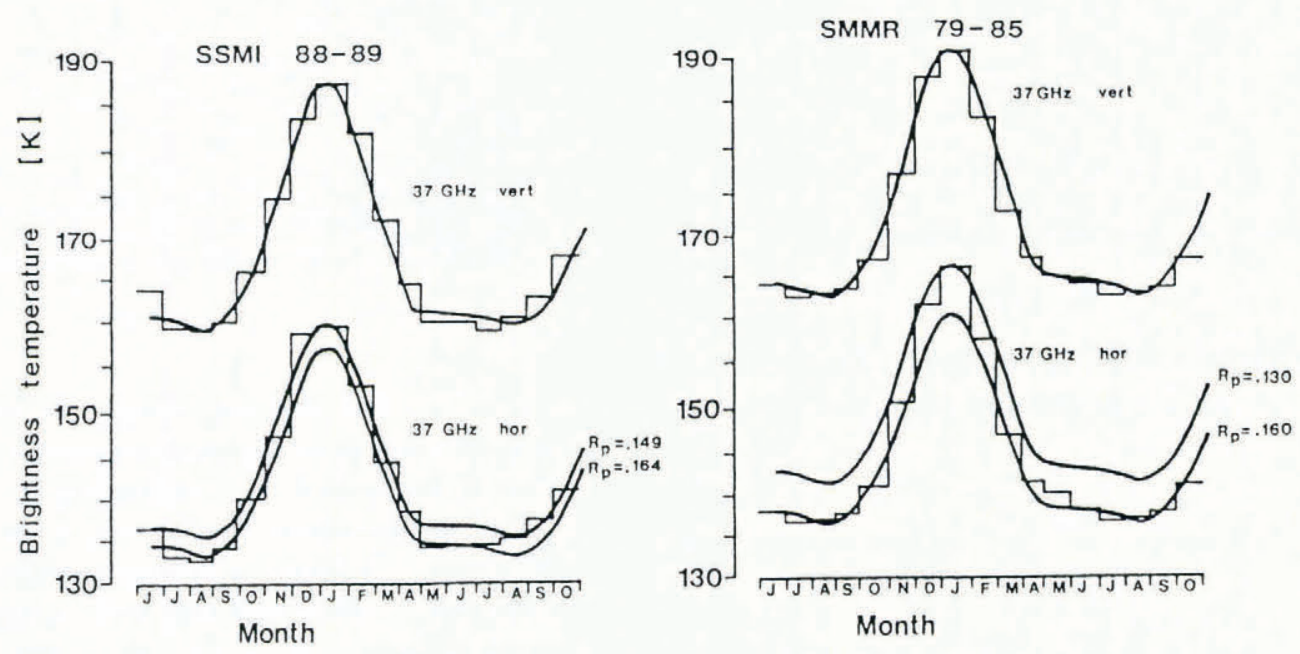

Fig. 4. As Figure 3 but for the $37 \mathrm{GHz}$ channels. 
Table 1. Radiative parameters determined by matching calculated and observed brightness temperatures. The last two columns give the scattering coefficient at the surface and the correction factor calculated from the modified radiative parameters, and using estimates for the absorption coefficient given in the literature
$\Gamma_{\mathrm{a}}$
$\Gamma_{\mathrm{so}}$
$\Gamma_{\mathrm{e}}$
$\gamma_{\mathrm{a}}$
$\gamma_{\mathrm{so}}$
$Z$
$\mathrm{m}^{-1}$
$\mathrm{m}^{-1}$
$\mathrm{dB} / \mathrm{m}$
$\mathrm{m}^{-1}$
$\mathrm{m}^{-1}$

$18 \mathrm{GHz}$ V

SMMR

\begin{abstract}
$37 \mathrm{GHz}$ V
\end{abstract}
SMMR

$19 \mathrm{GHz} \mathrm{V}$

SSMI

0.39

2.93

0.32

4.29

$37 \mathrm{GHz}$ V

SSMI
0.07

1.98

0.66

15.57

1.53

0.04

1.01

23.00

0.15

0.31

1.60

2.68

2.22

0.91

0.15

0.20

1.11

4.39

3.71

$37 \mathrm{GHz}$ vertical and horizontal channels covering a $100 \mathrm{~km}^{2}$ region around Vostok Station (central East Antarctica) are retrieved from the data set described in Jezek and others (1993). Daily values are averaged to obtain monthly mean brightness temperatures averaged over the region studied here for the period June 1988 to October 1989.

Figures 3 and 4 show the comparison between model calculations and observations. The upper panels show the measured brightness temperature for the vertical channels as well as the best model fit. For the vertical channels, the power reflection coefficient, $R_{\mathrm{p}}$, is near zero and model results were obtained for an observation angle of $49^{\circ}$ $\left(\Theta_{2}=32^{\circ}\right)$ using the values for the modified radiative parameters given in Table $1\left(s=0.015 \mathrm{~m}^{-1}\right)$.

Hallikainen and others (1987) measured an extinction coefficient of $3 \mathrm{~dB} \mathrm{~m}^{-1}$ at $18 \mathrm{GHz}$ and $7 \mathrm{~dB} \mathrm{~m}^{-1}$ at $37 \mathrm{GHz}$, for snow grains with a diameter of $0.5 \mathrm{~mm}$. Lytle and Jezek (in press) found an average loss of $3 \mathrm{~dB} \mathrm{~m}^{-1}$ through the Greenland firn when measured using a bandwidth ranging from 26.5 to $40.0 \mathrm{GHz}$. For the higher frequencies $(33.5-40.0 \mathrm{GHz})$ the measured losses average about $4 \mathrm{~dB} \mathrm{~m}^{-1}$, while for lower frequencies $(27-33.5 \mathrm{GHz})$ the average loss is about $2 \mathrm{~dB} \mathrm{~m}^{-1}$.

For the low-frequency channels (18 and $19 \mathrm{GHz}$ ), the values for the net loss near the surface found here are comparable to those measured by Hallikainen and others (1987) and Lytle and Jezek (in press). The net loss derived here for both $37 \mathrm{GHz}$ channels appears to be rather large and may not be fully realistic. The observed seasonal variation in $T_{\mathrm{B}}$ is large for this channel (about $28 \mathrm{~K}$ ) and the best model fit is very sensitive to uncertainties in this value and in the value of the minimum brightness temperature (Fig. 2). The temperature model uses long-term averaged climate data to calculate the firn temperature. However, it may very well be that the time period from June 1988 to October 1989 $\left(\mathrm{SSM} / \mathrm{I}\right.$ coverage; $\left.T_{\mathrm{Bmin}}=158.9 \mathrm{~K}\right)$ was preceded by a warm spell, so that the ice at depth is warmer than usual. Using observed SMMR brightness temperatures averaged over several years $\left(T_{\mathrm{Bmin}}=162.6 \mathrm{~K}\right)$ yields a more reliable estimate of the radiative parameters for the $37 \mathrm{GHz}$ channel. (For the $19 \mathrm{GHz}$ channel this problem is less of an issue because the seasonal amplitude is much smaller for this channel $(15 \mathrm{~K})$ and, as is evident from Figure 2, the best fit is much less sensitive to uncertainties in this value than is the case for the $37 \mathrm{GHz}$ channel.)

The lower panels in Figures 3 and 4 give the measured and calculated horizontal brightness temperature. For the horizontal channels, the power reflection coefficient is non-zero. In first approximation, horizontal brightness temperatures may be expected to be related to the vertical ones as

$$
T_{\mathrm{Bh}}=\left(1-R_{\mathrm{p}}\right) T_{\mathrm{Bv}} .
$$

However, as is clear from Figures 3 and 4, using this relation to calculate horizontal brightness temperatures yields a poor fit to the observed data, as well as requiring an unreasonably large power reflection coefficient. Using a constant power reflection coefficient, either the maximum or the minimum of observed brightness temperature can be modeled satisfactorily, but not the entire curve. This means that either the power reflection coefficient possesses seasonal variation (which seems very unlikely), or the scattering and absorption coefficients for the horizontal channel are different from those for the vertical channel. Another possibility is that the contribution from reflected sky energy to the brightness temperature needs to be included.

\section{COMPARISON OF RADIATIVE PARAMETERS WITH THEORY AND PREVIOUS STUDIES}

Zwally (1977) discussed at length how the radiative parameters describing absorption and scattering can be 
estimated from the Mie-Rayleigh scattering theory. For the firn at Plateau Station (central East Antarctica), he calculated $\gamma_{\mathrm{a}}=0.15 \mathrm{~m}^{-1}$ and $\gamma_{\mathrm{so}}=0.22 \mathrm{~m}^{-1}$ (for the $18 \mathrm{GHz}$ channel), corresponding to a net radiation loss of $1.6 \mathrm{~dB} \mathrm{~m}^{-1}$. The near-surface loss predicted by the present model $\left(1.53-1.98 \mathrm{~dB} \mathrm{~m}^{-1}\right)$ agrees with this theoretical value.

By comparing calculated emissivities with observed ones, Zwally (1977) finds that the theoretical scattering coefficient needs to be reduced by a factor 0.12 $\left(\gamma_{\mathrm{so}}=0.026 \mathrm{~m}^{-1}\right)$ to obtain agreement. Based on this, Zwally concludes that the Mie-Rayleigh theory overestimates scattering in polar firn. Comiso and others (1982) argue that the values for the radiative parameters used by Zwally (1977) result in a poor correlation between modeled and observed seasonal variation in brightness temperature. They find that $\gamma_{\mathrm{a}}=0.038 \mathrm{~m}^{-1}$ and $\gamma_{\mathrm{so}}=0.066 \mathrm{~m}^{-1}$ (i.e. a reduction factor of 0.3 ) yields a more realistic seasonal variation in $T_{\mathrm{B}}$ as well as a good agreement between calculated and observed emissivities. However, according to figure 5 in Comiso and others (1982), modeled summer brightness temperatures are about $5 \mathrm{~K}$ too low for Plateau Station, while for South Pole Station the modeled winter minimum is about $8 \mathrm{~K}$ too high. As shown in Figure 2, the radiative parameters strongly affect the winter minimum brightness temperature as well as the seasonal variation. Thus, the net radiation loss may have been underestimated by Comiso and others (1982).

In summary, Zwally (1977) and Comiso and others (1982) may have incorrectly partitioned energy loss between absorption and scattering. This could be because these authors use the emissivity as the primary matching parameter. However, the seasonal variation in brightness temperature is much more sensitive to changes in the radiative parameters than is the emissivity. Also, emissivity alone does not provide sufficient constraint to determine both $\gamma_{\mathrm{a}}$ and $\gamma_{\mathrm{so}}$, as pointed out by Comiso and others (1982).

Zwally (1977) used Equation (1) to calculate the brightness temperature. This expression is based on the approximation that neglects scattering as a radiative source term. If the same approximation is applied to the results obtained here (i.e. $Z=0$ and $\Gamma_{\text {so }}=\gamma_{\text {so }}$ ), similar results are obtained. With $Z=0$, the present model suggests that for the $18-19 \mathrm{GHz}$ vertical channel, $\gamma_{\mathrm{a}}=0.39-0.32 \mathrm{~m}^{-1}$ and $\gamma_{\mathrm{so}}=0.07-0.04 \mathrm{~m}^{-1}$. These values for the absorption coefficient are about twice the theoretical value, while the best-fit scattering coefficients are about one third to one fifth of the theoretical value. The net loss of radiation found here corresponds to what is predicted by the Mie-Rayleigh theory, but the partitioning between absorption and scattering does not agree with this theory. These results can be interpreted as showing the importance of the source term, $Z$, in the radiative transfer function. As noted earlier, the value of $Z$ does not affect the net radiation loss (Equation (6)) but it has a strong effect on the relative importance of absorption and scattering.

No estimates for the magnitude of the scattering source term are available. However, by adopting the value $\gamma_{\mathrm{a}}=0.15 \mathrm{~m}^{-1}(19 \mathrm{GHz} ; Z$ wally, 1977), and $\gamma_{\mathrm{a}}=0.91 \mathrm{~m}^{-1}(37 \mathrm{GHz}$; Hallikainen and others, 1987), the magnitude of the source term can be estimated, as well as the actual scattering coefficient (Equations (4) and (5)). The results are given in Table 1 .

The values for the near-surface scattering coefficients at 18 and $19 \mathrm{GHz}$ found here if the scattering source term is included, are comparable to the theoretical value $\left(0.22 \mathrm{~m}^{-1}\right)$. For all channels, scattering is now more important than absorption, as predicted by the theory. Thus, another explanation for the discrepancy between radiative parameters determined from a best model fit and those calculated from the Mie-Rayleigh scattering theory, as encountered by Zwally (1977), is that scattering as a radiation source is not negligible and should be included in the calculation of brightness temperature.

\section{EFFECTIVE TEMPERATURE}

For the long-term averaged SMMR data, the difference between brightness temperatures at different frequencies but like polarizations is maximum in summer and minimum in winter (Figs 3 and 4). Jezek and others (1990) suggest that this may be due to the asymmetric seasonal forcing. Because the summer is of short duration (two months), the warm temperature wave does not penetrate deeply into the firn. Hence, radiation originating from greater depths is more affected by the underlying cold temperatures than is the shallow-penetrating signal. This is true and the conclusion of Jezek and others (1990) that there will be less difference in effective physical
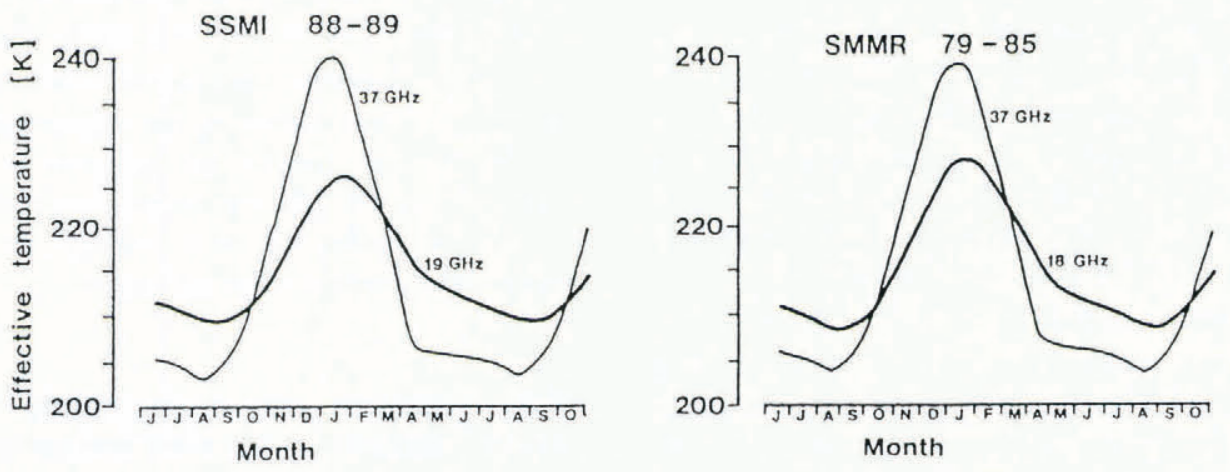

Fig. 5. Effective physical temperature of the firn for the 18 and 19 (heavy curves) and 37 (light curves) GHz vertical channels. 
temperature for the different channels during the winter months than during the summer, is confirmed by the present model.

The effective physical temperature $\langle T\rangle$, is the average physical temperature weighted by the radiative transfer properties at depth (Zwally, 1977) and normalized such that for an isothermal medium, $\langle T\rangle$ equals the physical temperature. In Figure 5 this quantity is shown for both vertical channels. The winter difference between the two SMMR temperatures is about $6 \mathrm{~K}$, while during the summer this difference increases to $13 \mathrm{~K}$. This is because during the summer months, a strong vertical temperature gradient exists, whereas during the winter differences between temperatures at various depths are less, thus giving a smaller difference in $\langle T\rangle$ for the two channels compared to the summer situation.

For the shorter record of SSM/I data, the situation is different. The low and high frequency brightness temperatures differ most during the austral winter, while the difference between the corresponding effective physical temperatures is largest in the summer. Because the effective physical temperature is normalized, and both the long-term and the short-term calculations are based on the same temperature profile, the two panels in Figure 5 could be expected to be similar. The fact that the observed records of brightness temperatures are different for the two data sets indicates that the period from June 1988 to October 1989 may have been preceded by a warmer than average period, resulting in higher temperatures at depth. This would mostly affect the deeper penetrating $19 \mathrm{GHz}$ channel and result in an above-average brightness temperature (Fig. 3).

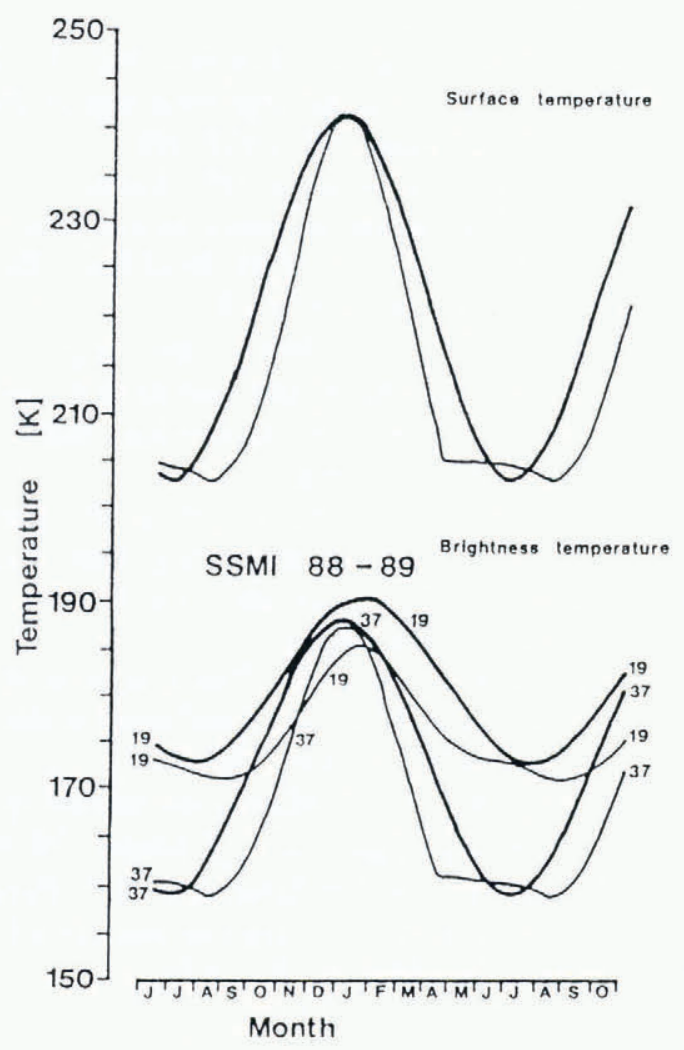

\section{SYMMETRIC FORCING}

Jezek and others (1990) raised the issue to what extent the asymmetric forcing affects brightness temperatures. In his calculations, Zwally (1977) prescribed the firn temperature at depth, choosing a symmetric time-dependent profile in which the summer and winter are of equal length. That is

$$
\begin{aligned}
& T(z, t)= \\
& 223.05-20.15 \exp [-0.3 z] \cos \left[0.99\left(t-t_{0}\right)-(97+20 z)\right]
\end{aligned}
$$

where $t$ represents time in days, with $t=t_{0}$ corresponding to the winter minimum. Figure 6 shows a comparison between brightness temperatures calculated using the actual firn temperature (Fig. 1) and those calculated using Zwally's parameterization (Equation (9)).

The $37 \mathrm{GHz}$ vertical channel originates from the near surface layer and consequently, the curve of brightness temperature closely reflects that of the surface temperature. Thus, other than the obvious differences (more symmetric curve of $T_{\mathrm{B}}$ ), prescribing the symmetric firn temperature yields results that are comparable to those obtained when the acțual climate data are used to calculate the firn temperature.

Differences are larger for the deeper penetrating 18 and $19 \mathrm{GHz}$ (vertical) channels. Using parameterization in Equation (9) results in an increase in winter minimum $T_{\mathrm{B}}$ of about $2 \mathrm{~K}$, and an increase in summer maximum of about $6 \mathrm{~K}$, compared to results discussed earlier. The reason for this is that the symmetric temperature profile over-estimates the temperature at depth during the austral winter (Fig. 7). More important, however, is that according to Equation (9) the summer and winter are of equal duration so that there is less winter cold that needs to be removed in the summer. This yields the largest increase in brightness temperature during the summer.

\section{CONCLUSIONS}

By including scattering as a radiative source term in the radiative transfer model developed by Zwally (1977),

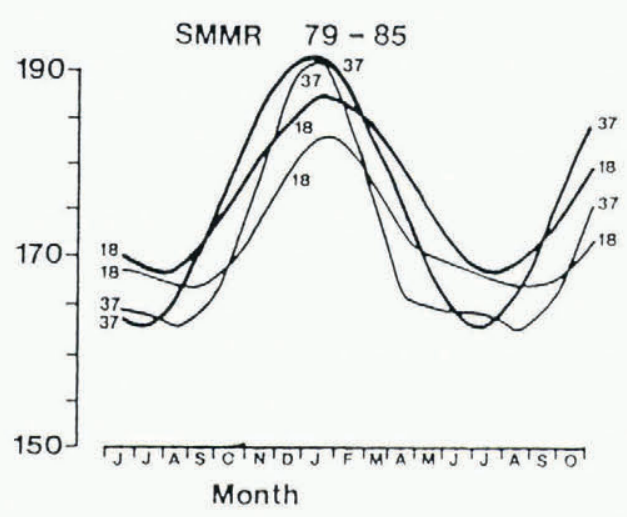

Fig. 6. Effect of symmetric temperature forcing on the brightness temperature. The upper panel shows the symmetric (heavy curve) and actual (light curve) surface temperature, and the lower panel the corresponding brightness temperatures for the 18-19 and $37 \mathrm{GHz}$ vertical channels. 


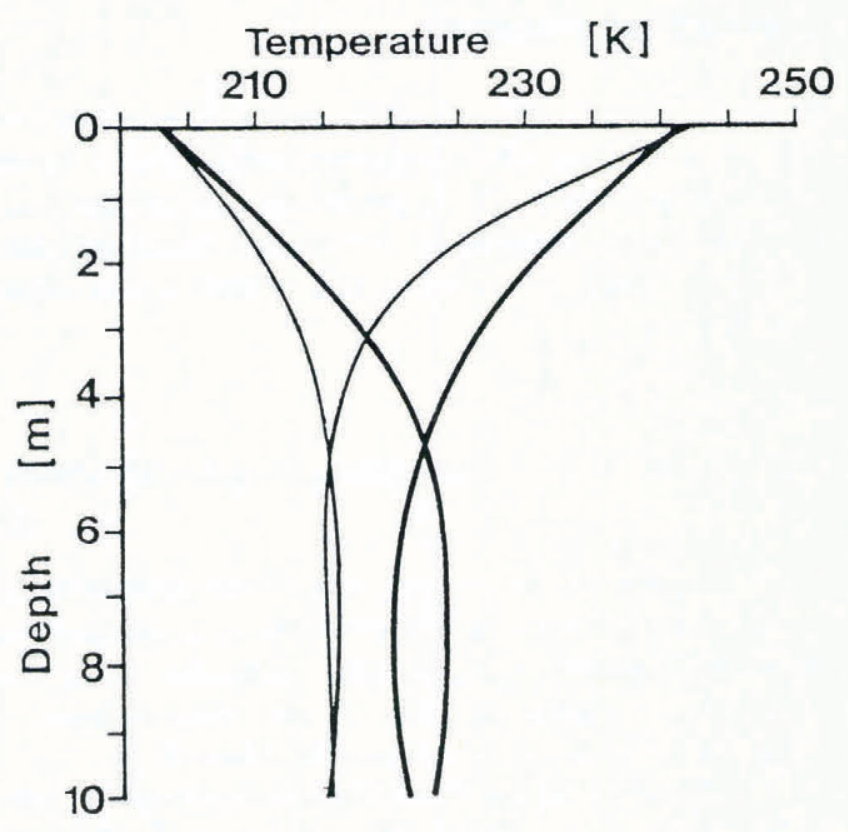

Fig. 7. Temperature profiles at the summer maximum and winter minimum according to parameterization (Equation (9)) (heavy curves) and calculated with the temperature model (light curves).

agreement between model-calculated radiative parameters and values determined from the Mie-Rayleigh scattering theory can be obtained. Neglecting this source term results in calculated absorption coefficients that are too large. Because the net radiation loss is independent of the scattering source term, this results in scattering coefficients that are too small. A more realistic partitioning between scattering and absorption is obtained when scattering as a radiation source is retained in the radiative transfer function.

By matching calculated brightness temperatures with observed ones, the radiative parameters can be determined unambiguously. This is not the case if emissivity is used as the sole tuning parameter. As pointed out by Comiso and others (1982), there may be more than one combination of radiative parameters that produce the observed emissivity, yet not all of these yield a realistic seasonal variation in brightness temperature.

The horizontal brightness temperature is not simply related to the vertical one through a constant power reflection coefficient. This indicates that both channels may have different radiative properties, or that the contribution from reflected sky energy to the brightness temperature may be important.

Symmetric versus actual temperature forcing has the largest effect on the deeper penetrating 18 and $19 \mathrm{GHz}$ brightness temperature. For the long-term record, the difference between brightness temperatures at different frequencies but like polarizations is largest in the summer and minimum in the winter, due to the asymmetrical seasonal forcing. During the summer months, the difference between the corresponding effective physical temperatures is largest.

By comparing records from individual years to average records spanning several years, inferences about variations in the local climate can be made.

\section{ACKNOWLEDGEMENTS}

J. Bolzan and H.J. Zwally are thanked for pointing out errors in an earlier version of the manuscript. This research was supported by the Polar Oceans Program at NASA Headquarters. Byrd Polar Research Center Contribution No. 809.

\section{REFERENCES}

Chang, T. C., P. Gloersen, T. Schmugge, T. T. Wilheit and H.J. Zwally. 1976. Microwave emission from snow and glacier ice. 7. Glaciol., 16(74), 23-39.

Chang, A. T. C., B. J. Choudhury and P. Gloersen. 1980. Microwave brightness of polar firn as measured by Nimbus 5 and 6 ESMR. 7. Glaciol., 25(91), 85-91.

Comiso, J.C., H.J. Zwally and J.L. Saba. 1982. Radiative transfer modeling of microwave emission and dependence on firn properties. Ann. Glaciol., 3, 54 58.

Greuell, W. and J. Oerlemans. 1986. Sensitivity studies with a mass balance model including temperature profile calculations inside the glacier. Z. Gletscherkd. Glazialgeol., 22(2), 101-124.

Greuell, W. and J. Oerlemans. 1989. The evolution of the englacial temperature distribution in the superimposed ice zone of a polar ice cap during a summer season. In Oerlemans, J., ed. Glacier fluctuations and climatic change. Dordrecht, Kluwer Academic Publishers, 289-303.

Hallikainen, M. T., F. T. Ulaby and T. E. van Deventer. 1987. Extinction behavior of dry snow in the 18- to 90 $\mathrm{GHz}$ range. IEEE Trans. Geosci. Remote Sensing, GE25(6), 737-745.

Jezek, K. C., D.J. Cavalieri and A. Hogan. 1990. Antarctic ice sheet brightness temperature variations. CRREL Monogr. 90-1, 217-223.

Jezek, K. C. and 6 others. 1991. Comparison between SMMR and SSM/I passive microwave data collected over the Antarctic ice sheet. Columbus, OH, Byrd Polar Research Center. (Technical Report 91-03.)

Jezek, K. C., C.J. Merry and D.J. Cavalieri. 1993. Comparison of SMMR and SSM/I passive microwave data collected over Antarctica. Ann. Glaciol., 17 (see paper in this volume).

Lytle, V.I. and K.C. Jezek. In press. Dielectric permittivity and scattering loss measurements of Greenland firn at 26.5 to $40 \mathrm{GHz}$. IEEE Trans. Geosci. Remote Sensing.

Schwerdtfeger, W. 1970. The climate of the Antarctic. In Landsberg, H. E., ed. World survey of climatology. Vol. 14. Amsterdam, Elsevier, 253-334.

Zwally, H.J. 1977. Microwave emissivity and accumulation rate of polar firn. J. Glaciol., 18(79), 195-215.

Zwally, H.J. and P. Gloersen. 1977. Passive microwave images of the polar regions and research applications. Polar Rec., 18(116), 431-450.

The accuracy of references in the text and in this list is the responsibility of the authors, to whom queries should be addressed. 